\title{
A DUAL FORM OF KURATOWSKI'S THEOREM
}

BY FRANK HARARY AND WILLIAM T. TUTTE

Communicated by J. W. Green, August 24, 1964

The celebrated criterion of Kuratowski [1] for the planarity of a graph $G$ asserts that $G$ is nonplanar if and only if it contains a subgraph homeomorphic to $K_{5}$ or $K_{3,3}$.
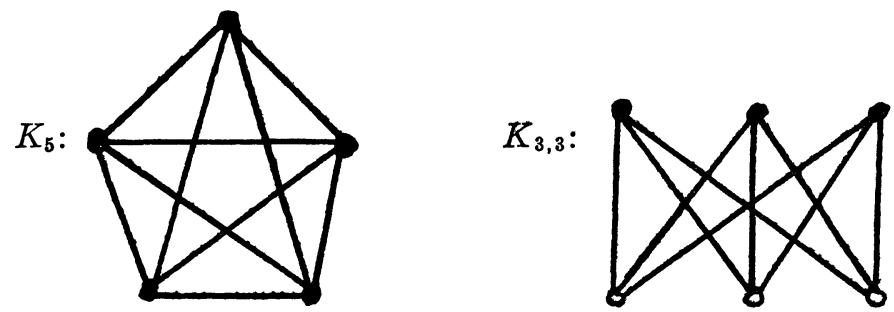

Whitney [3] proved that $G$ is planar if and only if it has a dual. Tutte [2] generalized the concept of duality from planar graphs (in which regions are replaced by vertices and common boundaries by edges) to general graphs by means of matroid theory.

It is surprising that the dual form of Kuratowski's Theorem has not been previously formulated. In practice we have found that this form is easier to apply, especially by using its corollary.

We say that a shrinking of $G$ is obtained when a connected subgraph of $G$ is replaced by a single vertex. A contraction of $G$ is the result of a sequence of shrinkings. A subcontraction of $G$ is a subgraph of a contraction. The dual form of Kuratowski's result is as follows.

TheOREM. $A$ graph is nonplanar if and only if it has $K_{5}$ or $K_{3, \mathbf{3}}$ as a subcontraction.

CoRollary. A connected graph is nonplanar if and only if it has $K_{5}$ or $K_{3,3}$ as a contraction.

The proofs will be given elsewhere.

\section{REFERENCES}

1. K. Kuratowski, Sur le problème des courbes gauches en topologie, Fund. Math. 15 (1930), 271-283.

2. W. T. Tutte, Matroids and graphs, Trans. Amer. Math. Soc. 90 (1959), 527-552.

3. H. Whitney, Non-separable and planar graphs, Trans. Amer. Math. Soc. 34 (1932), 339-362.

UNIVERSITY OF MICHIGAN AND

UNIVERSITY OF WATERLOO 